\title{
PARÂMETROS ESPAÇO-TEMPORAIS DO ANDAR EM CRIANÇAS OBESAS E COM PESO NORMAL DE ACORDO COM O SEXO
}

\section{SPACE-TEMPORAL PARAMETERS OF GAIT IN OBESE AND NON-OBESE CHILDREN ACCORDING TO THEIR SEX}

\author{
Ana F. R. Kleiner* \\ Lilian T. B. Gobbi* \\ Marcos T. P. Menucchi* \\ Maria D. T. de Oliveira* \\ Frederico Pieruccini-Faria*
}

Kleiner AFR, Gobbi LTB, Menucchi MRTP, Oliveira MDTd, Pieruccini-Faria F. Parâmetros espaço-temporais do andar em crianças obesas e com peso normal de acordo com o sexo. Rev Bras. Cresc. Desenv. Hum. 2004; 14(3): 29-36.

\begin{abstract}
Resumo: Características corporais interferem no desempenho motor. Para analisar os parâmetros espaço-temporais do andar em crianças obesas e de peso normal, por sexo, 23 crianças entre 7 e 10 anos de idade, foram intencionalmente selecionadas e distribuídas em dois grupos: obeso $(\mathrm{OB}, \mathrm{n}=9)$ e normal $(\mathrm{N}, \mathrm{n}=14)$. Marcadores reflexivos foram afixados em pontos anatómicos (quinto metatarso, maléolo lateral e calcando). Os participantes andaram sobre uma passarela de $8 \mathrm{~m}$ de comprimento na velocidade preferida e foram filmados no plano sagital. As variaveis descritivas analisadas foram: comprimento da passada (CP), comprimento da passada normalizado pela estatura (CPN) duração da passada (DP), velocidade da passada (VP), duração da fase de balanço (DFB) e duração da fase de suporte (DFS). Os resultados revelaram que: grupo isoladamente interfere em CPN e DP; sexo isoladamente afeta CP, CPN, VP; grupo e sexo afetam CPN, DFB, DP. Assim, o aumento da massa corporal ocasiona mudanças nos parâmetros espaço-temporais do andar quando sexo é considerado.
\end{abstract}

Palavras-chaves: Obesidade. Crianças. Andar. Parâmetros espaço-temporais.

\section{INTRODUÇÃO}

Atualmente, reconhece-se que a criança obesa corre sério risco de desenvolver diversas doenças em função da elevação dos valores sanguíneos de triglicérides e colesterol. Alterações ortopédicas (joelhos valgos, por exemplo), dermatológicas e respiratórias decorrentes da obesidade infantil tornam-se mais evidentes na vida adulta $^{1}$.

No Brasil, cinco em cada cem crianças de até 14 anos apresentam índices elevados de massa corporal ${ }^{1}$. Considerando as caractensticas da sociedade em que estes indivíduos estão inseridos, pode-se atribuir a incidência de obesidade em crianças a dois fatores interrelacionados: maior consumo de alimentos gordurosos (funk food e fast food) e uma vida sedentária².

A inatividad sica, aliada ao excesso de massa corporal, pode retardar o desenvolvimento motor da criança minimizando as possibilidades de experiências motoras necessárias para aquisição de habilidades básicas. A aquisição das habilidades motoras básicas é de grande importância, principalmente na primeira infância, pois é nesta fase que a criança adquire os movimentos moto-

Pesquisadores do Laboratório de Estudos da Postura e da Locomoção - LEPLO - Departamento de Educação Física/ Instituto de Biociências/Universidade Estadual Paulista - Av. 24-A, nº I 515 - Bela Vista 13.506-900 - Rio Claro/SP Fone: (19) 3526-4320 E-mail: leplo@rc.unesp.br - A/C da Professora Lilian T. B. Gobbi 
res fundamentais, que são a base para uma transição adequada para a aquisição de movimentos motores específicos ${ }^{3}$.

Leite $^{4}$, analisando os padrões fundamentais dos movimentos andar na trave e saltar horizontal, evidenciou que a massa corporal interferiu no comprimento médio da passada no andar na trave e na distância do saltar. O desempenho motor foi afetado pelas características dos indivíduos, entre elas a massa corporal.

Ferreira e Bohme ${ }^{5}$, em análise sobre a influência da adiposidade corporal e do sexo no desenvolvimento motor de crianças por meio de tarefas relacionadas ao deslocamento do corpo como um todo (testes de flexão na barra fixa para os membros superiores e salto em distância para os membros inferiores), constataram que a adiposidade corporal colaborou com um desempenho desfavorável nos testes, independente do sexo. Não foram encontradas diferenças estatisticamente significativas entre os sexos no desenvolvimento motor das crianças analisadas.

Quando a aquisição das habilidades motoras básicas não ocorre como o esperado, a criança poderá apresentar dificuldades em combinar e modificar estas habilidades de forma mais especializada, com nítida visualização de erros de execução ${ }^{6}$. Considerando que as habilidades motoras especializadas favorecem o engajamento da criança e do adolescente no convívio social, o relacionamento interpessoal da criança obesa pode também ser prejudicado.

$\mathrm{O}$ andar, mesmo parecendo uma habilidade simples e de fácil execução, apresenta um complexo relacionamento neuro-músculo-esquelético, especialmente quando terrenos irregulares estão presentes, como acontece na locomoção urbana. As características espaço-temporais do andar fornecem importantes informações acerca do padrão emergente frente às condições atuais da criança ${ }^{7,8}$.

Entendendo e assumindo que a emergência do padrão locomotor é o resultado da complexa relação do indivíduo, do ambiente e da tarefa ${ }^{9}$, este estudo preocupa-se com parâmetros do comportamento locomotor que revelem as alterações decorrentes da busca pelo sucesso da ação motora por um organismo que apresenta características diferenciados, tanto pela massa corporal como pelo sexo. Poucos são os estudos preocupados em observar a questão do sexo durante o desenvolvimento motor da criança e raros são os estudos direcionados a avaliar o andar de crianças obesas considerando o sexo.

Assim, o objetivo deste estudo foi analisar os parâmetros espaciais e temporais do andar em crianças obesas e com peso normal, considerando o sexo. Esperava-se que o acúmulo de adiposidade corpórea que, conseqüentemente, evidencia maior massa corporal, apresentasse diferenças, específicas para cada sexo, no padrão locomotor.

\section{MATERIAL E MÉTODO}

\section{Participantes}

Participaram deste estudo 23 crianças prépúberes, entre 7 e 10 anos de idade. A decisão da faixa etária dos participantes deveu-se a pouca influência de fatores orgânicos hormonais no desempenho motor, como por exemplo, o comprimento dos segmentos corporais. As crianças foram escolhidas intencionalmente, ou seja, selecionadas pelo experimentador em primeira instância visualmente e, depois, agrupadas de acordo com suas características antropométricas em: grupo obeso e grupo com peso normal (Tabela 1). Os responsáveis pelos participantes assinaram o termo de consentimento após serem informados de todos os procedimentos.

\section{Instrumentos para análise}

A formação dos grupos foi realizada por meio do Diagnóstico de Obesidade em Crianças e Adolescentes ${ }^{2}$, que calcula o índice de massa corpórea percentual (0,70 IMC), como segue:

$\%$ IMC $=\left(\right.$ peso/altura $\left.{ }^{2}\right) /\left(50^{\circ}\right.$ percentil para a idade peso/ $50^{\circ}$ percentil altura) X 100 .

Assim, considerou-se, para este estudo, que indivíduos com \% IMC abaixo de 1,10 como sendo com peso normal e indivíduos acima de 1,20 como obeso.

TABELA 1. Distribuição dos participantes por grupo $(G)$ e por sexo ( $M=$ masculino e $F$ = feminino); médias e desvios padrão da massa corporal (em quilogramas), \% IMC e estatura (em centímetro).

\begin{tabular}{|c|c|c|c|c|c|c|c|c|c|c|c|c|}
\hline \multirow[t]{2}{*}{ G } & \multirow[t]{2}{*}{$\mathbf{N}$} & \multicolumn{2}{|c|}{ N/Sexo } & \multicolumn{3}{|c|}{ Massa Corporal (kg) } & \multicolumn{3}{|c|}{ \% IMC } & \multicolumn{3}{|c|}{ Estatura (cm) } \\
\hline & & $\mathbf{M}$ & $\mathbf{F}$ & $\mathbf{M}$ & $\mathbf{F}$ & G & $\mathbf{M}$ & F & G & $\mathbf{M}$ & $\mathbf{F}$ & G \\
\hline \multirow[t]{3}{*}{ Normal } & 14 & 8 & 6 & 35,03 & 32,70 & 34,04 & 0,88 & 0,855 & 0,87 & 135,27 & 134,78 & 135,06 \\
\hline & & & & \pm & \pm & \pm & \pm & \pm & \pm & \pm & \pm & \pm \\
\hline & & & & 13,4 & 8,53 & 11,23 & 0,135 & 0,131 & 0,128 & 13,16 & 6,82 & 10,55 \\
\hline \multirow[t]{2}{*}{ Obeso } & 9 & 5 & 4 & 55,42 & 53,49 & 54,56 & 1,30 & 1,33 & 1,31 & 140,8 & 140,57 & 140,7 \\
\hline & & & & \pm & ${ }_{8.25}^{ \pm}$ & $8 \frac{ \pm}{63}$ & $\stackrel{ \pm}{ \pm}$ & $\stackrel{ \pm}{ \pm}$ & $\frac{ \pm}{0.067}$ & $4 \frac{ \pm}{20}$ & $\underset{6,30}{ \pm}$ & $\underset{4.87}{ \pm}$ \\
\hline Total & 23 & 13 & 10 & & & & & & & & & \\
\hline
\end{tabular}


Para a obtenção dos dados cinemáticos, marcadores reflexivos passivos (meia esfera de isopor medindo $2,5 \mathrm{~cm}$ de diâmetro e base de velcro) foram afixados sobre meias pretas, no pé direito dos participantes, nos seguintes pontos anatômicos: quinto metatarso, maléolo lateral da fíbula e face lateral do calcâneo.

\section{Procedimentos}

Inicialmente foram mensuradas a massa corporal e a estatura de cada participante por meio de uma balança antropométrica, com toeza, marca Filizola, com precisão de $100 \mathrm{~g}$ para a massa corporal e $0,1 \mathrm{~cm}$ para estatura.

Cada participante, individualmente, foi instruído a percorrer uma passarela livre (carpete emborrachado de $8 \mathrm{~m}$ de comprimento e 1,43 m de largura), em sua velocidade preferida, em 5 tentativas consecutivas.

Uma câmera de vídeo Panasonic (modelo NV - M 9000PN S-VHS) posicionada a aproximadamente 3 metros do participante filmou, a uma freqüência de aquisição de $30 \mathrm{~Hz}$, no plano sagital direito da passarela, três ciclos completos de andar. Um ciclo completo (compreende os eventos entre um toque do calcanhar direito até o próximo toque do calcanhar direito), central e perpendicular à câmera, de cada tentativa, foi selecionado para análise. As imagens foram posteriormente capturadas pelo programa Microsoft [R] Vidcap (Video Capture Tool) em formato AVI (áudio vídeo interlace). Para o desentrelaçamento, calibração e medição das imagens foi utilizado o software Dvideow - versão 4.0 (Laboratório de Instrumentação Biomecânica da UNICAMP ${ }^{10}$ ).

A calibração das imagens foi realizada com uma cruz (de 1 m de distância entre as extremidades), que foi filmada por 5 segundos antes do início da coleta dos dados de cada participante. Desta forma, foi possível gerar as coordenadas bidimensionais (X: sentido antero-posterior, horizontal; e Y: vertical). Este procedimento fornece medidas conhecidas para que, através do software Dvideow, os dados medidos em pixel fossem transformados em medidas reais conhecidas (cm).

\section{Análise dos dados}

Os dados do deslocamento dos marcadores passivos foram filtrados usando o filtro Butterworth de $4^{\text {a }}$ Ordem com uma freqüência de corte de $5 \mathrm{~Hz}^{11}$. As variáveis dependentes medidas foram:

a. Comprimento da passada (CP): medida pela diferença dos valores da coordenada X referente aos momentos de toque do calcanhar (TC) direito e próximo TC direito, expresso em centímetros; b. Duração da passada (DP): medida pelo número total de quadros entre o momento do TC direito e o próximo TC direito, multiplicado por 0,033 e expressa em segundos;

c. Duração da fase de balanço (DFB): medida pelo número total de quadros entre o momento da retirada dos dedos do pé direito do chão até o momento do TC direito subseqüente, multiplicado por 0,033 e expressa em segundos;

d. Duração da fase de suporte (DFS): medida pelo número total de quadros entre o momento do TC direito até o momento da retirada dos dedos do mesmo pé, multiplicado por 0,033 e expressa em segundos;

e. Velocidade média da passada (VP): medida pela divisão dos valores do comprimento da passada pela duração de cada passada e expressa em metros por segundo.

ANOVAs com dois fatores grupo (obeso e normal) e sexo, por tentativa, foram empregadas para determinação das diferenças entre os fatores para cada variável dependente. Em todas as análises estatísticas foi adotado o nível de significância alfa de 0,05 .

\section{RESULTADOS}

Os resultados são apresentados de acordo com o parâmetro analisado, ou seja, iniciase pela variável espacial seguida pelas variáveis temporais e encerrando com a variável espaço-temporal.

A. Variável Espacial (comprimento da passada - CP): a ANOVA para dois fatores (grupo X sexo) revelou apenas efeito principal de sexo (F, “ $0=7,001$; p c 0,01; Figura 1). Os participantes do sexo masculino, independente do grupo, apresentaram maior CP $(1,15 \mathrm{~m})$ do que os indivíduos do sexo feminino $(1,09 \mathrm{~m})$.

Figura 1. Representação gráfica das médias e desvios padrão do $\mathrm{CP}$ por sexo ( $\mathrm{M}$ = masculino e $\mathrm{F}=$ feminino)

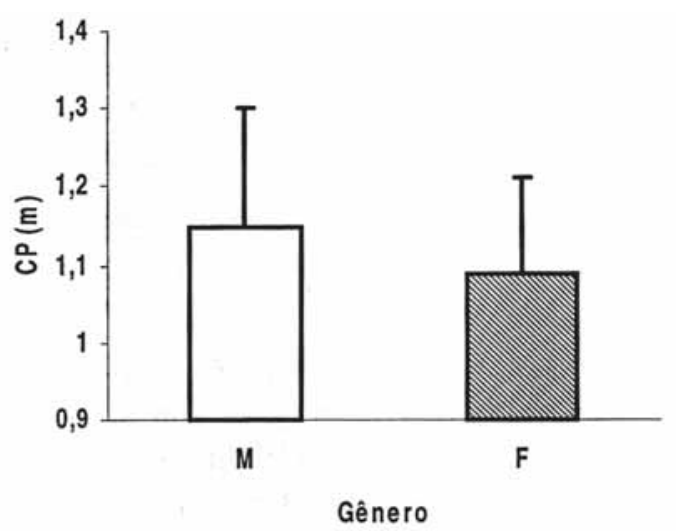


Considerando que CP é influenciado pela estatura do participante ${ }^{11}$, os dados de CP foram normalizados por esta variável. Após este procedimento, a ANOVA indicou, para a variável comprimento da passada normalizado (CPN, CP/estatura), efeitos principais de sexo $\left(\mathrm{F}_{1,110}=9,84 ; \mathrm{p}<0,003\right.$; Figura 2a) e de grupo $\left(\mathrm{F}_{1,110}^{1,110}=6,47 ; \mathrm{p}<0,013\right.$; Figura $\left.2 \mathrm{~b}\right)$ e interação, marginalmente significativa, entre grupo e sexo $\left(F_{1,110}=3,84 ; p=0,052 ;\right.$ Figura 2c). Destaca-se que o grupo obeso feminino apresenta CPN menor que os demais grupos, não havendo alteração nos grupos do sexo masculino.

B - Variáveis Temporais: duração da fase de balanço (DFB), duração da fase de suporte (DFS) e duração da passada (DP)

A ANOVA não acusou efeito principal ou interação da variável DFS com as variaveis independentes grupo e sexo.

Figura 2. Representação gráfica dos valores médios e de desvios padrão do CPN por sexo (a), por grupo (b) e interação entre sexo e grupo (c)
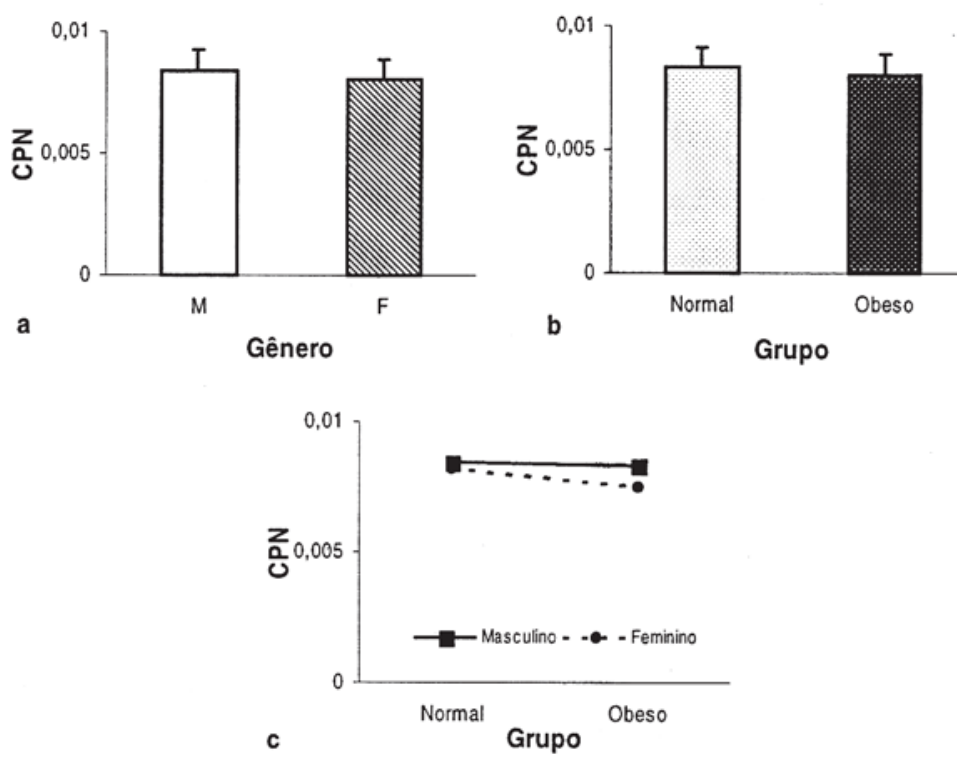

B.l - DFB: a ANOVA para dois fatores (grupo $\mathrm{X}$ sexo) revelou interação entre grupo e sexo $\left(\mathrm{F}_{1,110}=24,84\right.$; $\mathrm{p}<0,001$; Figura 3). Os participantes masculinos obesos apresentaram menor DFB que seus pares com peso normal, enquanto que para o sexo feminino aconteceu o inverso.

B.2 DP: a ANOVA revelou efeito principal de grupo $\left(\mathrm{F}_{1,110}=3,968 ; \mathrm{p}<0,05\right.$; Figura $\left.4 \mathrm{a}\right)$ e

Figura 3. Representação gráfica da interação entre grupo e sexo durante DFB.

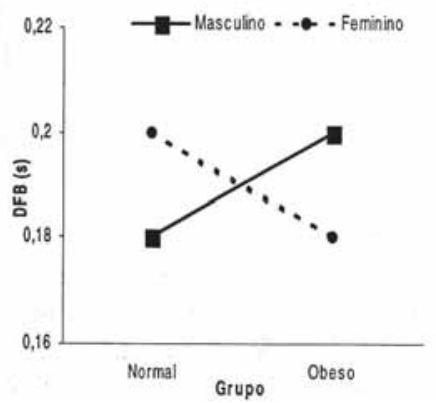

interação entre grupo e sexo $\left(\mathrm{F}_{110}=16,61\right.$; $\mathrm{p}<0,001$; Figura 4b). Por meio da Figura 4b, pode-se observar que as diferenças quanto ao grupo na DP podem ser atribuidas aos participantes do sexo masculino.

C - Variável Espaço-Temporal (VP): a ANOVA revelou apenas efeito principal de sexo $\left(\mathrm{F}_{1,110}=10,46\right.$; $\mathrm{p}<0,003$; Figura 5), evidenciando que os participantes do sexo feminino apresentam uma menor VP.

Figura 4. Representação gráfica dos valores médios e de desvios padrão do DP por grupo (a) e
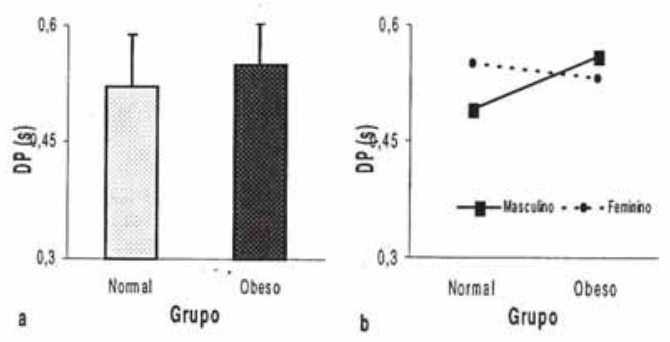
Figura 5. Representação gráfica dos valores médios e de desvios padrão da VP por sexo ( $\mathrm{M}=$ masculino e $\mathrm{F}$ = feminino)

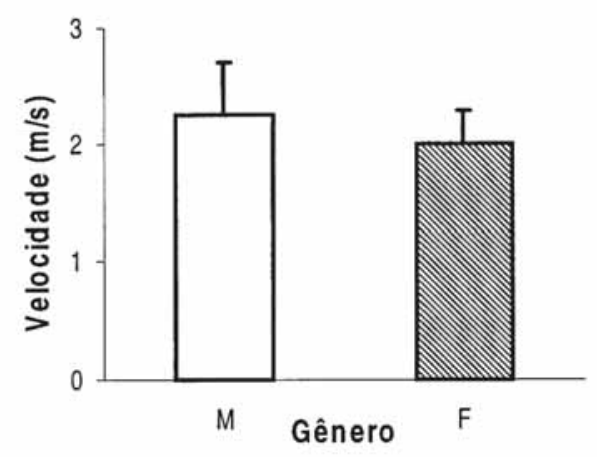

\section{DISCUSSÃO}

O presente estudo foi realizado com o objetivo de analisar os parâmetros espaciais e temporais do andar em crianças obesas e com peso normal, considerando o sexo. Esperávamos que o acúmulo de adiposidade que, conseqüentemente, evidencia maior massa corporal, apresentasse diferenças, específicas para cada sexo, no padrão locomotor. Os resultados evidenciaram que algumas diferenças nos parâmetros espaço-temporais do andar são específicas da massa corporal, outras do sexo e outras da relação entre obesidade e sexo. Desta forma, a discussão dos resultados trata primeiramente das diferenças quanto à obesidade, seguida pelos efeitos do sexo e completa-se com a relação entre eles nos parâmetros espaçotemporais do padrão locomotor de crianças.

\section{Obesidade}

A aquisição de habilidades motoras fundamentais maduras (manipulação, locomoção e equilíbrio) não é dependente da idade, mas de fatores relacionados à tarefa, ao indivíduo e ao ambiente $^{13,9}$. A obesidade apresenta-se como um aspecto do fator relacionado ao indivíduo. Desta forma, a obesidade deve estar interferindo na aquisição das habilidades motoras fundamentais seja pelo excesso de massa corporal, pela inatividade física ou pela associação de ambos.

Estudos têm evidenciado que o excesso de adiposidade corporal causa alterações nos parâmetros cinemáticos e temporais da marcha em crianças $^{13,14} \mathrm{e}$ em adultos e idosos ${ }^{15,16}$. Os resultados deste estudo, em crianças, evidenciaram que a obesidade, independente do sexo, afeta a duração e o comprimento da passada, quando este é normalizado pela estatura.

McGraw e col. ${ }^{14}$ analisaram os parâmetros cinemáticos da marcha, em três condições de ca- dência, e o equilíbrio de crianças do sexo masculino (entre 8 e 10 anos), obesas e não obesas e constataram que os indivíduos obesos apresentaram maiores valores nas DFB e duração da fase de duplo suporte, independente das condições impostas. Os resultados obtidos neste estudo não revelaram efeito principal de grupo para DFB, DFS e VP, não confirmando os resultados obtidos por McGraw e col. ${ }^{14}$. Entretanto, cabe considerar que o presente estudo envolveu também indivíduos do sexo feminino. Outra possibilidade é associar a DFB com o CPN, o que implica na utilização de uma estratégia adaptativa. Para manter o comprimento da passada em níveis similares a seus pares com peso normal, os indivíduos obesos aumentará a DFB. Esta estratégia facilita aos obesos acompanharem seus pares, mas representa uma sobrecarga sobre a perna de suporte e aumenta a demanda de controle do equilíbrio. Mesmo adotando esta estratégia, ocorreu diferença estatisticamente significativa na DP, ou seja, os indivíduos obesos utilizaram maior tempo para realizar um ciclo de passada em relação ao grupo com peso normal.

Applegate e col. ${ }^{15}$, por meio de análise cinemática, compararam a marcha de um adulto jovem não obeso com um obeso, constatando que o participante não obeso ocupou $55 \%$ do tempo total do movimento de marcha com a fase de oscilação, enquanto o jovem obeso ocupou 52\%, indicando que o indivíduo obeso apresentou maior tempo na fase de suporte.

Spyropoulos e col. ${ }^{16}$ compararam o padrão locomotor de adultos obesos adultos de peso normal e verificaram que os obesos apresentaram os menores valores médios de DP, o que foi atribuído à menor cadência e ao menor comprimento da passada apresentado pelos obesos. Em contrapartida, no estudo de Applegate e col. ${ }^{15}$, verificou-se que na DP o tempo foi similar entre os dois participantes sendo o tempo total da DP no jovem não obeso de 1,12 s e do jovem obeso 1,16 s. Hills e colo citam que, durante a marcha, as mais importantes articulações das extremidades inferiores ficam expostas a uma carga considerável. Quando isso ocorre, a absorção da força de reação na articulação é de, aproximadamente, três a cinco vezes a massa corporal e influencia nos parâmetros temporais do andar. Entretanto, o presente estudo não revelou diferenças na DFS entre os grupos. Cabe destacar que os estudos de Hills e col. ${ }^{17}$ e Applegate e colo foram realizados com indivíduos adultos jovens, no qual o medo de quedas e as diferenças estruturais orgânicas geram forças ativas e passivas diferentes, sendo estes alguns dos fatores determinantes nas alterações do padrão locomotor relacionadas à estabilidade. 
Em geral, indivíduos obesos têm tendência a andar em menor velocidade quando comparados a indivíduos não obesos, o que pode ser atribuído ao excesso de tecido adiposo entre as coxas, pouca resistência à fadiga e ao aumento da base de suporte ${ }^{10}$. Hills e Parker ${ }^{18}$ mostraram que crianças pré-púberes obesas apresentam uma maior duração da passada, uma menor velocidade, menor cadência e maior duração da fase de suporte. Os resultados do presente estudo não corroboram os descritas por Spyropoulos e col. ${ }^{16}$ e Hills e Parker $^{18}$, pois revelaram não haver diferença estatisticamente significativa entre os grupos para velocidade média da passada e duração da fase de suporte. Entretanto, outras diferenças foram observadas quanto ao sexo e que são discutidas a seguir.

\section{Sexo}

O acúmulo de tecido adiposo pode ocorrer em diferentes regiões corporais e induzir adaptações locomotores diferentes. Quando o tecido adiposo encontra-se concentrado na região abdominal (o que é mais comum entre homens), ocorre um deslocamento do centro de massa para frente, que é compensado pelo deslocamento do tronco e ombros para trás. Quando a adiposidade apresenta-se concentrada na região da pelve e coxas (mais comum em mulheres), ocorre um aumento da base de suporte para evitar o contato entre as coxas. No primeiro caso, uma alteração locomotora esperada é a diminuição no comprimento da passada, enquanto que no segundo caso, espera-se aumento da duração da fase de duplo suporte e da duração da passada. Os resultados revelaram diferenças no comprimento da passada e na velocidade média.

Na análise do CP e do CPN, foi observada diferença em relação ao sexo, sendo que os meninos apresentaram valores maiores nas duas variáveis. Durante os anos de infância e adolescência, diferenças quanto ao sexo vêm sendo encontradas no desempenho de diversas tarefas motoras. Machado e col. ${ }^{6}$, em estudo sobre a relação entre composição corporal e a performance de padrões motores em escolares (driblar, receber, rebater uma bola com bastão, chutar e arremessar), observaram que os meninos apresentam resultados significativamente superiores em relação às meninas. De modo geral, esta pequena vantagem observada no sexo masculino é fato atribuído parcialmente às diferenças estruturais”, fisiológicas ${ }^{19}$ e à presença de fatores biológicos e culturais ${ }^{20}$.

Em relação aos fatores biológicos, Machado e col. ${ }^{6}$, ao analisarem estatura e massa corporal de 80 crianças (40 de cada sexo), constataram que os meninos possuem maior valor dc massa magra e menor percentual de gordura corporal quando comparados com as meninas. Este fato pode estar demonstrando que as meninas podem estar iniciando as transições estruturais do início da fase de maturação sexual. Talvez, com a obesidade, estas diferenças apresentem-se acentuadas, gerando efeitos diferentes nas adaptações locomotoras.

Para a velocidade, os meninos apresentaram maior VP em relação às meninas. Alves e Gonçalves ${ }^{13}$, analisando o efeito da velocidade na força de reação do solo em crianças obesas com 10 anos de idade, também constataram que existem diferenças significativas no padrão da marcha entre meninos e meninas independente dc serem obesos.

\section{Obesidade $\mathrm{X}$ sexo}

Os resultados deste estudo revelaram uma associação entre obesidade e sexo no CPN, na DFB e na DP.

O grupo obeso feminino apresentou valores médios de CPN menores em relação a todos os outros grupos. Desde o nascimento, as meninas apresentam uma camada maior de tecido adiposo sob a pele ${ }^{21}$ e possuem também maior tendência à obesidade ginóide ou obesidade tipo $\mathrm{III}^{22}$. Neste tipo de obesidade, a concentração de massa gorda se localiza na região Isquio-fibular, o que pode gerar redução no CPN das meninas.

Para o grupo normal feminino foram evidenciados maiores valores médios de DP, quando comparado ao grupo feminino obeso e maior DFB em relação aos demais grupos. De forma especulativa, estes resultados podem ser atribuídos ao uso demasiado do quadril durante a marcha. Foi observado, durante a coleta dos dados, que algumas participantes executaram a marcha simulando o andar em uma passarela, ou seja, com excessivo deslocamento médiolateral da pelve. Frigo e col..$^{23}$ observaram, em 18 jovens mulheres, o movimento do tronco durante a marcha e constataram que todos os segmentos analisados do tronco apresentaram um ângulo de $5^{\circ}$, exceto o ângulo entre os ombros e a pelve que apresentaram uma curvatura proximal no plano frontal. O mesmo não aconteceu com as meninas obesas talvez pelo estereótipo físico da manequim profissional.

O grupo obeso masculino apresentou uma maior DP quando comparado aos outros grupos, em especial ao obeso feminino. Isto se deve à estratégia adotada para a realização da tarefa, ou seja, os meninos obesos aumentaram o comprimento da passada e a duração da fase de duplo suporte, o que reflete diretamente na DP. 
Estudos futuros devem endereçar questões associando outras medidas de adiposidade além da \% IMC, como dobras cutâneas por região corporal, circunferências e perímetros. Considerando que uma das características do obeso é a presença de joelhos valgos, sugere-se também a observação da rotação interna do calcâneo. Todos estes aspectos podem influenciar o comportamento locomotor destes indivíduos.

Os resultados obtidos neste estudo permitem concluir que a massa corporal aumentada, isoladamente, e sua associação com o sexo alteram os parâmetros espaço-temporais do andar em crianças obesas, indicando que estratégias locomotores adaptativas foram empregadas.
Crianças obesas e não obesas, e de sexo diferentes, desenvolvem estratégias locomotoras próprias para garantir a realização desta ação motora, adaptando-se às alterações estruturais referentes às restrições do organismo e favorecendo a estabilidade do sistema locomotor.

\section{AGRADECIMENTOS}

Os autores são gratos à Márcia Valéria Cozzani, por sua colaboração na captura das imagens; as crianças e seus responsáveis, pela participação; e à escola, por gentilmente ter cedido o espaço.

\begin{abstract}
Considering that body features interfere in motor performance, the aim of this study was to analyze the changes in spatial and temporal parameters of gait in obese, and non-obese children according to the gender.23 children intentionally selected participated in this study and they were distributed between two experimental groups according to their body mass index: obese (OB, $n=9)$ and normal $(N, n=14)$. Ali the participants had written consent forms signed by their parents. Reflexive markers were attached on the following body landmarks of the right foot: fifth metatarsal, malleolus lateral and calcaneus. The participants were asked to walk on an $8 \mathrm{~m}$ rubber pathway at a preferred pace while their locomotor behavior were recorded using a video camera Panasonic (model NV - M9OOOPN S-VHS) in a sagittal plane. Using a MatLab routine, the following variables were collected: stride length (SL), stride length normalized by height (SLN), stride duration (SD), stride velocity (SV), swing phase duration (SPD), and support phase duration (SUD). Data was statically analyzed using ANOVA with groups and gender as factors. Results revealed that: group alone interfere on SLN and SD; gender alone affects SL, SLN and SV; group and gender affects SLN, SPD and SD. These results allowed us to conclude that the increase in body weight can make changes in spatial and temporal parameters of children gait when gender is considered.
\end{abstract}

Key-words: Obesity. Children. Gait. Space-temporal parameters.

\section{REFERÊNCIAS}

1 Kaufman A. Obesidade infantojuvenil. Pediatria Moderna 1999; 32(4): 218-22.

2 Halpern A, Mancini MC. Obesidade. Revista Brasileira de Medicina 2000; 57:77-86.

3 Tani G, Manoel EJ, Kokubun E, Proença JE. Edu cação ffsica escolar: fundamentos de uma abor dagem desenvolvimentista. São Paulo: EPU/ EDUSP; 1988.

4 Leite HSF. Crescimento somático e padrões fundamentais de movimento: um estudo em escola res [dissertação]. Rio Claro (SP): Instituto de Bio ciências da UNESP; 2002. 5.

5 Ferreira M, Bohme MTS. Diferenças sexuais no desempenho motor de crianças: influência da adiposidade corporal. Revista Paulista de Educação Física 1998; 12(2): 181 -92.

6 Machado H, Campos W, Silva S. Relação entre composição corporal e a performance de padrões motores fundamentais em escolares. Atividade Física c Saúde 2002; 7(1): 63-70.
7 Sutherland D. The development of mature gait. Gaitand Posture 1997; 6: 163-70. 8.

8 Sutherland DH, Olshen RA, Biden E. N.; Wyatt, M.P. The development of mature walking. Oxford: Blackwell; 1988.

9 Newell KM. Constraints on the development of coordination. In: Wade MG, Whiting W, editors. Motor development in children: aspects of coordination and control. Dordrecht: Martinus Nijho ff; 1986. p.341-60.

10 Barros RML, Brenziokofer R, Leite N. J, Figueroa P. Desenvolvimento e avaliação de um sistema de análise cinemática tridimensional de movimen tos humanos. Revista Brasileira de Engenharia Biomédica 1999; 15 (1/ 2): 79-86.

11 Winter DA. Biomechanics and motor control of human movement. $2^{\mathrm{a}}$ ed. New York: WileyInters cience; 1990.

12 Gallahue DL, Ozmun D. Compreendendo o de senvolvimento motor: bebês, crianças, adolescentes e adultos. São Paulo: Phorte; 2001. 
13 Alves MGS, Gonçalves CA. Análise da marcha em diferentes velocidades: estudo em crianças obesas. In: Anais do IX Congresso Brasileiro de Biomecanica; 2001, maio 29 jun.01; Gramado. Gramado: UFRGS; 2001. p.218-23.

14 McGraw B, McClenaghan BA, Williams HG, Dickerson J, Ward DS. Gait and postural stability in obese and nonobese prepubertal boys. Archives of Physical and Medical Rehabilitation 2000; 81: 484-9.

15. Applegate K, Bassett C, Smith K. The gait of an obese vs. a non-obese college aged male. Moti on Analysis Projects, 2004. Disponível em: URL: http://www.umich.edu/mvs 330/ w99/obesity/main.html, acesso em 28/04/ 2004.

16 Spyropoulos P, Pisciotta JC, Konstantin P, Cairns MA, Simon SR. Biomechanical gait analysis in obese men. Archives of Physical and Medical Rehabilitation 1991;72: 1065-70.

17 Hills AP, Henning EM, Byrne N. M. Steele JR. The biomechanics of adiposity - structural and func tional limitations of obesity and implications for movement. Obesity Reviews 2002; 3(1): 35-43.
18 Hills AP, Parker AW. Gait characteristics of obese children. Archives of Physical and Medical Rehabilitation 1991; 72: 403-7.

19 Herkowitz J. Sex-role expectations and motor behavior of the young child. In: Ridenour M.V, editor. Motor development: issues and applications. Princeton: Princeton Book; 1978. p.83-98.

20 Hensley LD, East WB, Stiulwell JL. Body fatness and motor performance during preadolescence. Research Quarterly for Exercise and Sport 1982; 53(2): 113-40.

21 Bee H. A criança em desenvolvimento. São Paulo: Artmed; 1985.

22 Guedes D, Guedes J. Controle do peso corporal: composição corporal, atividade física $e$ nutrição. Londrina: Midiograf; 1998.

23 Frigo C, Carabalona R, Dalla Mura M, Negrini S. The upper body segmental movements during walking by young females. Clinical Biomechanics 2003; 18: 419-25.

Recebido em 24/06/2004

Modificado em 19/08/2004

Apovado em 23/08/2004 